\title{
Group psychoeducation for stabilised bipolar disorders: 5-year outcome of a randomised clinical trial
}

F. Colom, E. Vieta, J. Sánchez-Moreno, R. Palomino-Otiniano, M. Reinares, J. M. Goikolea, A. Benabarre and A. Martínez-Arán

\section{Background}

The long-term efficacy of psychological interventions for bipolar disorders has not been tested.

\section{Aims}

This study assessed the efficacy of group psychoeducation to prevent recurrences and to reduce time spent ill for people with bipolar disorders.

\section{Method}

A randomised controlled trial with masked outcome assessment comparing group psychoeducation and nonstructured group intervention during 5-year follow-up. One hundred and twenty people with bipolar disorders were included in the study and 99 completed 5-year follow-up. Time to any recurrence, number of recurrences, total number of days spent ill, frequency and length of hospitalisations were the main outcome measures.

\section{Results}

At the 5-year follow-up, time to any recurrence was longer for the psychoeducation group (log rank=9.953, $P<0.002)$. The psychoeducation group had fewer recurrences (3.86 v. 8.37, $F=23.6, P<0.0001$ ) of any type and they spent less time acutely ill (154 V. 586 days, $F=31.66$, $P=0.0001$ ). The median number of days of hospitalisation per hospitalised participant was also lower for the psychoeducation group (45 v. 30, F=4.26, $P=0.047$ ).

\section{Conclusions}

Six-month group psychoeducation has long-lasting prophylactic effects in individuals with bipolar disorders. Group psychoeducation is the first psychological intervention showing such a long-term maintained efficacy in people with bipolar disorders.

\section{Declaration of interest}

E.V. has served as consultant, advisor or speaker for the following companies: Astrazeneca, Bial, Bristol-Myers, Eli Lilly, Glaxo-Smith-Kline, Jannssen-Cilag, Lundbeck, Merck-Sharp and Dohme, Novartis, Organon, Otsuka, Pfizer Inc, SanofiAventis, Servier and UCB Pharmaceuticals. F.C. has served as advisory or speaker for the following companies: Astra Zeneca, Eli-Lilly, Sanof-Aventis, Tecnifar and Shire. Funding detailed in Acknowledgements.
The evidence for the use of psychoeducation and family-focused therapy as prophylactic adjuncts to medication in people with stabilised bipolar disorders is, at present, quite conclusive. ${ }^{1,2}$ Our group, in 2003, published a randomised controlled study of recurrence prevention using group psychoeducation, reporting efficacy of this intervention in preventing mania and hypomania, depression and mixed episodes, decreasing the rates of rehospitalisation $^{3}$ and enhancing adherence. ${ }^{4}$ Several psychosocial approaches have shown different degrees of efficacy in the short term. ${ }^{5-8}$ All the cited studies examined the efficacy of several psychological interventions at a maximum of 2-year follow-up. However, there are no data on the longer-term maintenance efficacy of such treatments.

The purpose of the present article is to report an additional 3 years of follow-up for our original trial, resulting in a total of 5-year follow-up. Moreover, we report data on time spent acutely ill, a very relevant issue regarding functioning and quality of life. ${ }^{5,6}$ To our knowledge, this is the first randomised masked clinical trial assessing the longer-term efficacy of a psychological intervention as a prophylactic adjunct to pharmacotherapy in bipolar disorders.

\section{Method}

\section{Study design}

This is a continuation study of a published randomised clinical trial. ${ }^{3}$ The first part of the study had a 2-year cut-off but in the present study we have extended the assessments up to 5 years. This was a parallel two group (experimental and control) randomised, single-masked trial with 20 weeks of treatment, carried out in the Bipolar Disorders Program of the Hospital Clinic of Barcelona (Barcelona, Spain), whose research and ethics committee approved the study. This programme merges clinical care, research and education, and focuses primarily on people with the most severe bipolar I and II disorders.

The study had two phases. The treatment phase consisted of 21 weeks of randomised treatment in which all participants received standard psychiatric care with standard pharmacological treatment. The experimental group $(n=60)$ received additional group psychoeducation (21 sessions on a weekly basis) and the control group ( $n=60)$ met every week in groups of $8-12$ without special instructions from the therapist (21 meetings on a weekly basis). This design was aimed to control the variability induced by the possible supportive effect of the group reunions themselves. To avoid interferences, participants agreed not to visit any psychologist outside the centre.

The follow-up phase comprised 5 years during which all participants continued receiving standard pharmacological treatment without psychological intervention in the study centre and were assessed monthly for several outcome measures. For statistical purposes we considered the whole time of the study after randomisation (5 years and 6 months) in our follow-up. After written informed consent was signed, participants were assessed at baseline and randomised, stratifying the groups by gender, age and number of previous episodes. Randomisation 
was made by a computerised random number generator, ensuring restricted randomisation allocation and was run by an independent statistician. All the statistical analysis was run considering as a start the point where the intervention began. Significance was set at $P=0.05$.

\section{Treatment}

\section{Standard psychiatric care}

All participants were seen by two psychiatrists (E.V. or A.B.) every 4 weeks and were specifically told to go to the centre whenever they felt any change in their mood or any other problem such as insomnia. The psychiatrists had a minimum of 4 years of clinical and research experience in bipolar disorders.

Participants received pharmacological treatment following the treatment algorithms of the Barcelona Bipolar Disorders Program. Psychiatrists and assessors were masked to the nature of the treatment given to the participants, who were told not to tell their psychiatrists if they were receiving psychoeducative treatment. The psychiatrist was not allowed to provide formal psychotherapy or specific psychoeducation beyond good clinical practice.

\section{Participants}

One hundred and twenty people fulfilling DSM-IV ${ }^{9}$ criteria for bipolar disorder type I or II, aged 18-65 years, were recruited from participants with bipolar I and II disorders enrolled in the naturalistic prospective follow-up of the Bipolar Disorders Program of the Hospital Clinic at the University of Barcelona (Spain). Inclusion criteria were a lifetime diagnosis of bipolar disorder type I or II elicited by a trained psychiatrist (E.V. or A.B.); being euthymic (Young Mania Rating Scale (YMRS) ${ }^{10}$ score $<6$, Hamilton Rating Scale for Depression (HRSD-17) ${ }^{11}$ score $<8$ ) for at least 6 months immediately prior to study entry; having sufficient data on the prior course of illness collected from a prospective follow-up of at least 24 months; and written consent to participate in the study. Exclusion criteria were current DSM-IV Axis I comorbidity (only if severe conditions and excluding caffeine and nicotine dependence) assessed by means of the Structured Clinical Interview for DSM-IV Axis I Disorders (SCID-I; 'mental retardation' (IQ < 70); organic brain damage; or deafness). Individuals currently receiving any kind of psychotherapy or enrolled in any pharmacological trial were also excluded. Axis II comorbidity (except mental retardation) was not considered as an exclusion criteria.

\section{Psychoeducation group}

Group psychoeducation was performed according to our manual. ${ }^{13}$ This programme consists on 21 sessions of $90 \mathrm{~min}$, each aimed at improving four main issues: illness awareness, treatment adherence, early detection of prodromal symptoms and recurrences and lifestyle regularity. The programme was in groups of $8-12$, conducted by two experienced psychologists (F.C. and A.M.-A.) who had previous experience with bipolar patients (3 years) and specific training on patients' group management. The structure of each session consisted of a 30-40 min talk on the topic of the day, followed by an exercise related to the issue (e.g. drawing a life chart, writing a list of potential triggering factors) and a discussion. Participation was encouraged.

\section{Control group}

In addition to standard pharmacological treatment, participants assigned to the control group received an intervention consisting of 20 weekly group meetings of 8-12 participants with the same two psychologists (F.C. and A.M.-A.). The psychologists had instructions to avoid giving any psychoeducational feedback.
Examples of this non-psychoeducative attitude could be answers like 'What do you think?' or 'What is the group opinion towards this comment?' when a question was posed to the therapist.

\section{Assessments}

All participants were assessed monthly by the study psychiatrists and every 2 weeks by a research assistant who had instructions to contact the psychiatrist if a recurrence was suspected. The psychiatrist and research assistant were masked to treatment and participants were requested not to reveal significant details.

All participants in this study had been enrolled in the naturalistic prospective follow-up of the Bipolar Disorders Program of Barcelona for at least 2 years. This follow-up includes assessment of recurrences, symptom checking and treatment registration, and is performed every 2 months. Baseline assessment includes the administration of the SCID-I, SCID-II, YMRS, HRSD-17, and the Holmes \& Rahe inventory for stressful lifeevents, which are also repeated every 2 months or whenever a new episode was suspected by the psychiatrist in charge of the participant.

Psychiatric medication and reasons for changes were recorded. The number of hospitalisations, reasons for admission and the total number of days that the participant remained hospitalised were also recorded. Adherence was assessed by the combination of an adherence-focused interview with the individual, an adherence-focused interview with significant first-degree relatives or a partner and by analyses of plasma concentrations of mood stabilisers. This method has been extensively explained elsewhere. ${ }^{8}$

\section{Main outcome measures}

\section{Time to recurrence}

The primary outcome measure was time to recurrence. This was defined as the time (days) elapsed between baseline and the emergence of a new acute episode according to DSM-IV criteria and scores above or equal to 20 on the YMRS for manic recurrence; above or equal to 12 for hypomanic recurrence; above or equal to 17 on the HRSD-17 for depressive recurrence; and above or equal to 20 on the YMRS and 12 on the HRSD-17 for mixed recurrence.

\section{Number of recurrences}

The number of recurrences, separating for type of episode (manic, hypomanic, mixed or depressive) was also recorded as a secondary outcome measure.

\section{Time spent ill}

Another secondary outcome measure was time spent ill. We obtained this data by prospectively registering the number of days that a participant fulfilled criteria for a specific episode.

\section{Participant flow and drop out}

Overall, 120 euthymic out-patients were recruited at the Bipolar Disorders Program of Barcelona; 60 were randomly assigned to psychoeducation and 60 to the control group. Group rules, which were provided equally to both experimental and control participants, included the possibility of group exclusion for those individuals missing five sessions. Within the experimental group, 44 individuals out of $60(73.3 \%)$ fully adhered to the psychoeducation programme. Sixteen people $(26.6 \%)$ withdrew from the group for a number of reasons, the most common being manic recurrence (eight participants) followed by depressive recurrence (four participants) emerging during group intervention. Full participant flow is shown in Fig. 1. 


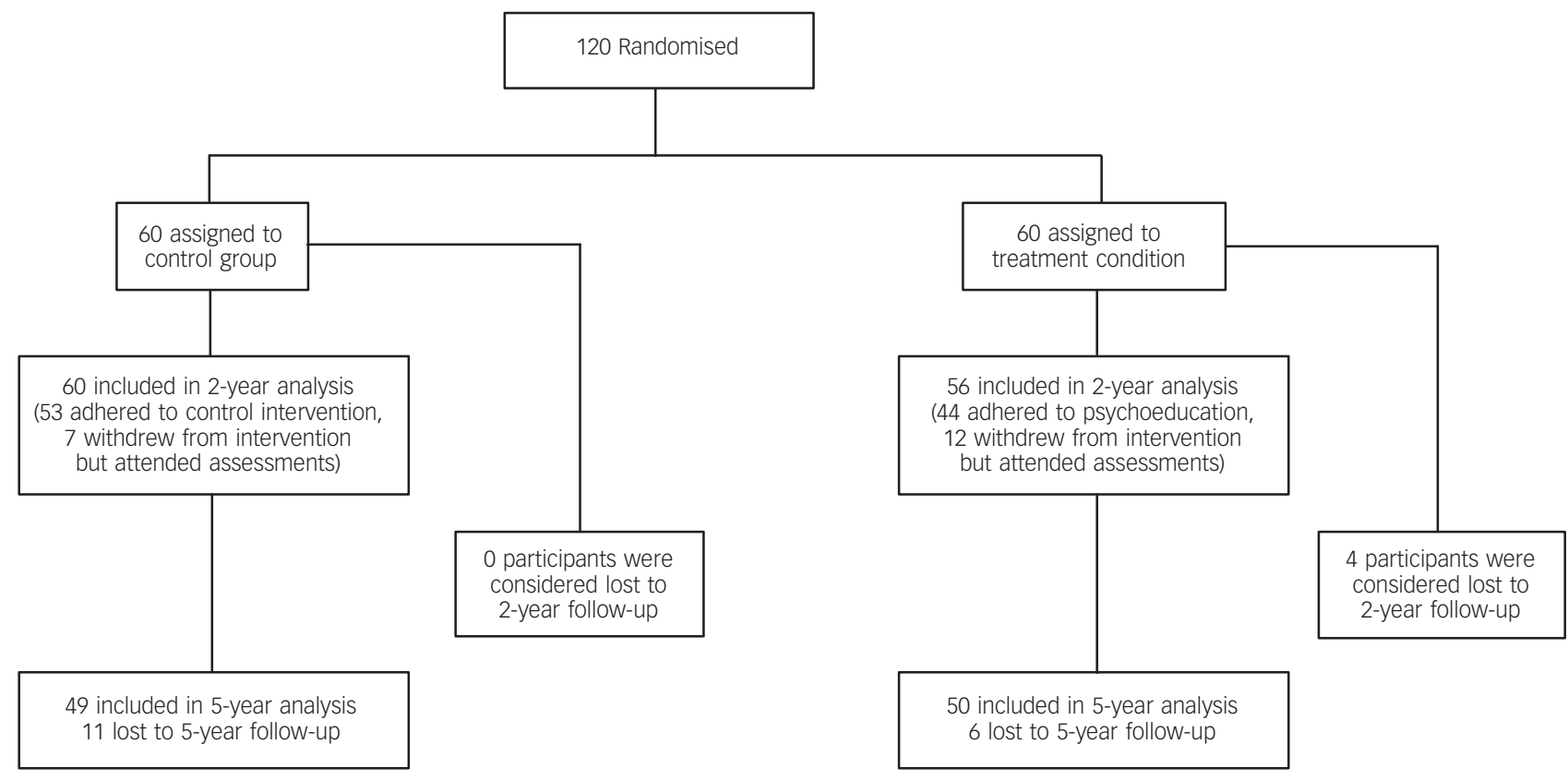

Fig. 1 CONSORT flow of participants

Out of 60 people in the control group, $7(11.6 \%)$ withdrew from the weekly meetings but continued with the psychiatrist visits, the pharmacological treatment and the follow-up phase procedures. The total number of participants who withdrew was higher among the experimental group $(P<0.05)$. There were no significant differences in the number of sessions not attended: people in the control group did not attend a mean of 3.75 (s.d.=0.87) sessions compared with 3.38 (s.d.=1.16) sessions in the treatment group.

Twenty-one participants out of 120 (25.2\%) dropped out from the 5-year follow-up and had to be considered as lost to follow-up. Of these, 11 belonged to the control group and 10 to the psychoeducation group. Hence, the number of non-completers per group was almost equal.

\section{Baseline characteristics of treatment groups}

Both groups were comparable at baseline regarding clinical (Table 1) and therapeutic variables. Each group consisted of 38 women and 22 men with a similar number of previous episodes.

\section{Statistical analysis}

Recurrence free curve analysis was performed using KaplanMeyer's survival analysis. Cox proportional hazards regression analysis was used to assess the association between number of previous episodes and time to recurrence, independent of other predictors. The following covariates were included in every analysis: age at onset, number of previous episodes and number of previous hospitalisations. Comparison of baseline characteristics of the sample were made by a $\chi^{2}$-test for categorical variables such as gender, diagnostic subtype, polarity of first episode, history of rapid cycling, seasonal pattern, psychotic features, history of suicide attempts, Axis II comorbidity and type of treatment received using the Fisher $z$-test when needed and the $t$-test for quantitative variables. The separate analysis at follow-up of the number of participants who relapsed for each condition was by a $\chi^{2}$-test. The comparison of the mean number of recurrences during the treatment and the follow-up phase was made using the ANCOVA model including age at onset, number of previous episodes and number of previous hospitalisations as covariates. Statistical significance was set at $P<0.05$.

\begin{tabular}{|c|c|c|c|}
\hline Characteristic & $\begin{array}{l}\text { Control group } \\
\qquad(n=60)\end{array}$ & $\begin{array}{l}\text { Psychoeducation group } \\
\qquad(n=60)\end{array}$ & Statistical test ${ }^{a}$ (d.f.) \\
\hline Male gender, $n(\%)$ & $22(36.7)$ & $22(36.7)$ & $Z=0(1)$ \\
\hline Diagnostic subtype, bipolar I: n (\%) & $48(80)$ & $52(86.7)$ & $z=0.96(1)$ \\
\hline Psychotic features, yes: ${ }^{\mathrm{b}} n(\%)$ & $42(70)$ & $47(78.3)$ & $Z=1.08(1)$ \\
\hline Attempted suicide, yes: ${ }^{b} n$ (\%) & $19(31.7)$ & $21(35)$ & $Z=0.15(1)$ \\
\hline Axis II comorbidity, yes: n (\%) & $22(36.7)$ & $15(25)$ & $Z=1.91(1)$ \\
\hline Age, years: mean (s.d.) & $34.26(7.80)$ & $34.03(9.32)$ & $t=0.15$ \\
\hline Age at onset, years: mean (s.d.) & $23.25(7.55)$ & $22.26(6.69)$ & $t=0.75$ \\
\hline Total number of episodes, mean (s.d.) & $8.81(6.60)$ & $10.31(10.55)$ & $t=-0.93$ \\
\hline Number of hospitalisations, mean (s.d.) & $2.01(2.12)$ & $1.81(1.78)$ & $t=0.56$ \\
\hline
\end{tabular}




\section{Results}

\section{Time to recurrence}

Survival analysis of participants remaining in remission is shown in Fig. 2. The event curves for the control and treatment groups were significantly different for time to any recurrence (log rank=9.953, $P<0.002)$. Number of previous episodes was not associated with time to recurrence (95\% CI 0.013-0.461, $P=0.49$ by Cox model)

\section{Number of recurrences}

Over the 5-year follow-up period, people in the psychoeducation group had less recurrences than those in the non-psychoeducation group (3.86 v. 8.37, $F=23.6, P<0.0001$ ). When analysing each type of episode (mania, hypomania, mixed or depression), individuals in the psychoeducation group had a lower number of recurrences. When comparing effect sizes (Cohen's $d$ ) between the 2-year and the 5-year assessments, we could see how effect sizes did not decrease with the passing of time. This was true for any episode $(0.79 v .0 .87)$, mania $(0.40 v .0 .57)$, hypomania $(0.27 v .0 .42)$ and mixed episodes $(0.34 v .0 .61)$. As for depression, the effect size at 5 years was slightly inferior to the 2-year effect size but still in the range of large effect $(0.91 v .0 .80)$.

\section{Time spent ill}

People in the psychoeducation group spent much less time acutely ill than those in the non-psychoeducation group. This is mainly owing to the dramatic differences accounting for time spent with depression, but it is also true for every type of episode (Table 2).

\section{Hospitalisations}

During the treatment phase, 8 individuals out of $60(13.3 \%)$ in the control group were hospitalised owing to recurrence compared with 9 out of $60(16.1 \%)$ in the treatment group (non-significant difference). At the end of the 5-year follow-up period, 24 individuals out of $60(40 \%)$ in the control group had been

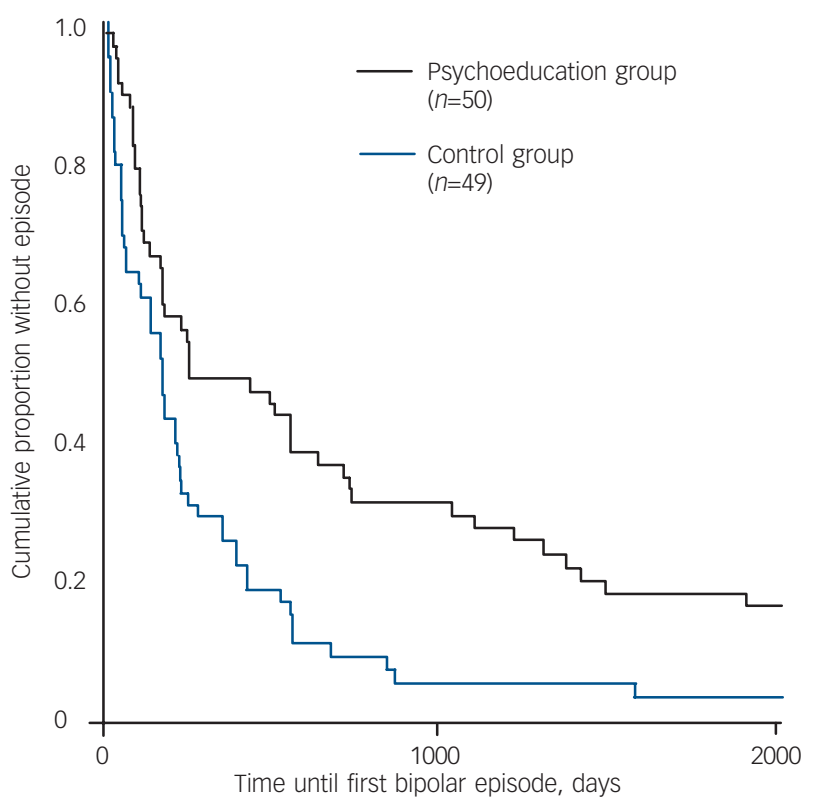

Fig. 2 Survival curve on time to recurrence. hospitalised v. 17 out of $56(30.4 \%)$ in the treatment group $(P=0.278)$.

The number of hospitalisations at the 5 -year follow up for the psychoeducation group was 0.24 (s.d. $=0.52)$ admissions $v .0 .59$ (s.d.=0.96) in the control group $(F=5.34, P=0.023)$. The median number of days of hospitalisation per hospitalised participant was also lower for people in the psychoeducation group (45 v. 30) $(F=4.26, P=0.047)$.

\section{Treatment adherence}

As for treatment adherence, both groups were comparable at entry, with rates of poor adherence reaching $25.9 \%$ ( 15 individuals out of 58) in the control group and 30.5\% (18 individuals out of $59)$ in the psychoeducation group at baseline $\left(\chi^{2}=0.312, P=0.577\right)$. At 5-year follow-up there were no differences regarding adherence, with rates of poor adherence of $14.3 \%$ and $14 \%$ respectively $\left(\chi^{2}=0.002, P=0.967\right)$.

\section{Discussion}

Although several psychological interventions tested on people with bipolar disorders report short-term prophylactic efficacy (up to 1 year), the use of 6-month group psychoeducation is the first one to show efficacy in the long term. In fact, other psychological therapies may lose effect in the middle term. This may be the case for cognitive-behavioural relapse prevention therapy as this intervention showed prophylactic efficacy in the short term, 1 year, ${ }^{5}$ but had no significant effect in relapse reduction after 2 years other than the number of days being ill. $^{16}$ Thus, the present study might change the way in which psychological interventions for bipolar disorders are considered, as one of the traditional criticisms that psychotherapy for severe mental disorders has received in the past is that its effect may progressively disappear once the intervention has finished. In contrast, our study shows how a time-limited, single intervention, without the help of any booster session, is able to bring about a major improvement in the outcome of bipolar disorders in the long term (5 years). Thus, group psychoeducation may enhance behavioural and attitudinal changes that seem to be maintained over the years.

The study has several limitations. Generalisability is limited by the exclusion of individuals with severe comorbidities and substance misuse, and by the fact that this was a single-site trial. The outcome was measured as the presence of full episodes and subthreshold presentations were not included in the definition. Masking may also be an issue in this sort of study, as some participants might have made comments to the psychiatrists that would indirectly indicate their treatment allocation.

All our 5-year follow-up data confirm the 2-year follow-up findings: the number of bipolar episodes is much lower for the psychoeducation group over time. In the long term, group psychoeducation may prevent all sorts of episodes including mania. Interestingly enough, when comparing 2-year and 5-year follow-up, prevention of mania seems to be more powerful in the longer term (Cohen's $d$ effect size $=0.4 v .0 .57$ ), probably as a result of the fact that changes concerning regularity of habits and, especially, early detection, may be more noticeable in the longer term. Psychoeducation goes far beyond a mere transmission of information and should be considered as diseasemanagement training more than anything else. According to this view, it is completely logical that its effects are more noticeable with the passing of time (Cohen's $d$ effect size for prevention of all sorts of episodes $=0.79$ v. 0.87). 
Table 2 Comparison of mean number of days spent on each episode at 5-year follow-up

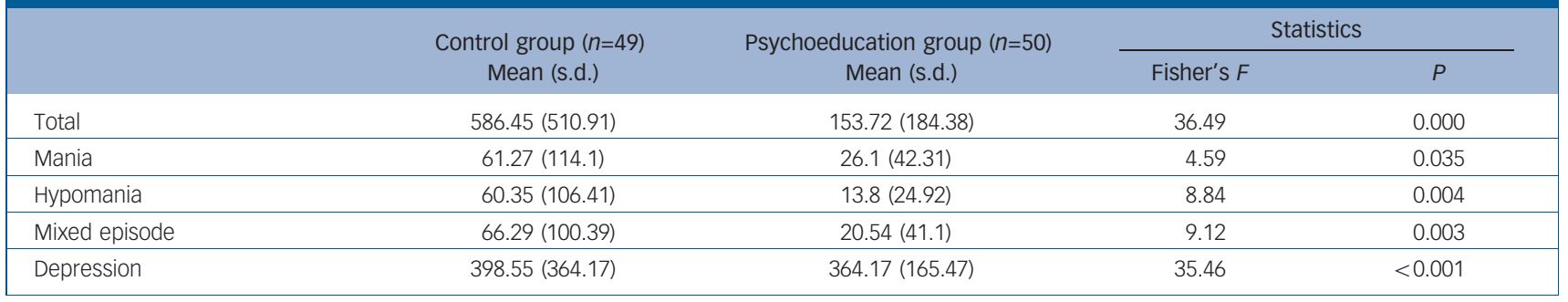

On the other hand, it should be emphasised that the psychoeducation programme and the subsequent follow-up was highly accepted by our participants, as most of them did not withdraw and engaged in the follow-up visits, as demonstrated by the fact that just $20 \%$ of the participants had to be considered as lost to follow-up at the 5-year end-point.

It is worth mentioning the effect of psychoeducation on hospitalisations. We could not find any difference regarding the number of participants who required hospitalisation, either at 2- or at 5-year follow-up. However, a significant difference was found regarding the number of hospitalisations per individual, lower for people in the psychoeducation group, which may mean that through psychoeducation we could be avoiding the 'revolving door' phenomena (the frequent rehospitalisation of a subgroup of people). This effect has not been described by any other psychological intervention in bipolar disorders. Simon et $a l^{8}$ reported how their 'systematic care program' for bipolar disorders had a significant effect on reducing the frequency and severity of mania at a worthy cost but could not find any efficacy on hospitalisations.

We have observed a significant effect of group psychoeducation on time spent ill, whereas individuals in the nonpsychoeducation group spent up to $30 \%$ of their time acutely ill; for people in the psychoeducation group, this was approximately just $8 \%$. Results for the control group were slightly different from those reported by Judd et al ${ }^{17,18}$ as people in that study spent between $47 \%$ and $54 \%$ of time being ill; depending on the bipolar subtype, this difference was somewhat predictable if we keep in mind that we were using quite narrower recurrence criteria. But what is more striking is the benefit regarding time spent in each sort of episode depending on the participation or not in a psychoeducation programme: the psychoeducation group participants spent $66 \%$ less time in mania, hypomania or mixed episodes and spent $75 \%$ less time in depression. Again, psychoeducation seems to have more effect on days in an episode than other interventions that previously looked at this issue: participants assigned to cognitive therapy spent $35 \%$ less time in mania and $56 \%$ less time in depression. ${ }^{16}$

Regarding the lack of effect of psychoeducation on adherence, it might well be a statistical artefact related to selective withdrawal and lack of data of those less adherent individuals in both groups, giving the false idea of both groups 'improving' their adherence rates. An alternative hypothesis though would be that the effect of the group on adherence disappears with the passing of time. In this case, booster sessions would be highly recommended.

Further research on the use of psychoeducation should look at the specific mechanisms by which group psychoeducation reduced every single type of episode. According to the study of Perry et al, ${ }^{19}$ prodromal sign identification would be especially efficacious for preventing mania but not depression. Improvement of adherence is another obvious potential mechanism ${ }^{20}$ as people on lithium showed significantly more stable lithium levels after psychoeducation than the control group. ${ }^{4}$ Our own previous studies ${ }^{21}$ showed the efficacy of psychoeducation with people who adhered. Hence, prophylaxis would not rely exclusively on adherence enhancement, although this is a very important part of the programme. A further potential mechanism of psychoeducation might be regularity of habits, particularly regarding sleep; insomnia may either be a prodromal sign of recurrence (which can be targeted with early recognition) and a triggering factor for an episode; sleep deprivation has been reported to induce manic switch, ${ }^{22}$ and interventions specifically (but not exclusively) aimed at improving sleep have been proven to be effective in people with bipolar disorders. ${ }^{23}$ Moreover, another potential active ingredient of psychoeducation may be the improvement of comorbidities, and specifically Axis II features. Although individuals with acute substance misuse or dependence were not enrolled, one session was devoted to potential risks of substance misuse, including legal drugs such as alcohol or caffeine, which may have a detrimental effect on sleep. Furthermore, in a secondary analysis of 2-year data, people with comorbid Axis II disorders significantly improved with psychoeducation. ${ }^{24}$ In a recent trial, cognitivebehavioural therapy was not effective in preventing further episodes in people with bipolar disorders in general, and particularly in those with comorbidities. ${ }^{25}$ The improved understanding about the disease and increase of illness awareness may be another factor underlying the positive effects of psychoeducation, especially regarding empowerment for decision-taking, which may include dose increase of certain medications as soon as some warning sign is detected, if the psychiatrist is not available at that point. ${ }^{13}$ The better the knowledge about the disease is, the lower the burden on the individual and caregivers. ${ }^{26}$ The decrease in subjective burden might correlate with increased resilience and perhaps changes in brain neuroplasticity, providing a biological background for the mechanism of action of this psychological intervention. Increased behavioural resilience may help people to cope with stigma and this might account for some of the large effects on time spent in depression in this study. Although some of these mechanisms may be still somewhat speculative, they are clearly related to the content of the psychoeducational package that was delivered to the participants.

No treatment is free of side-effects and a few people had some kind of undesired behavioural reactions to psychoeducation. Namely, three individuals reported 'increased anxiety', 'fear' or 'ruminations'. Another person started to obsessively check his mood and reported mood changes day in day out during a 3-week period after the session devoted to mood checking. Most of these changes were transient, but they deserve careful monitoring; too often, psychotherapies are seen as free of adverse events and this is simply not true. However, this is the first time that a particular form of psychotherapy is proved to be effective in the real long term (up to 5 years) in bipolar disorders, significantly reducing illness morbidity and recurrence rates. 
F. Colom, PsyD, MSc, PhD, Bipolar Disorders Program, Institute of Neurosciences, Hospital Clinic, IDIBAPS, CIBER-SAM, University of Barcelona, Spain, and Psychologica Medicine Division, Institute of Psychiatry, London, UK; E. Vieta, MD, PhD, J. Sánchez-Moreno, PSyD, Bipolar Disorders Program, Institute of Neurosciences, Hospital Clinic, IDIBAPS, CIBER-SAM, University of Barcelona, Spain

R. Palomino-Otiniano, PsyD, Department of Psychiatry, Oxford University, Oxford, UK; M. Reinares, PsyD, J. M. Goikolea, MD, A. Benabarre, MD, PhD, A. Martínez-Arán, PSyD, MSC, PhD, Bipolar Disorders Program, Institute of Neurosciences, Hospital Clinic, IDIBAPS, University of Barcelona, Spain.

Correspondence: E. Vieta, Bipolar Disorders Program, Hospital Clinic of Barcelona, Villarroel 170, 08036 Barcelona, Spain. Email: EVIETA@clinic.ub.es

First received 21 May 2007, final revision 26 Feb 2008, accepted 18 Mar 2008

\section{Acknowledgements}

This work was partly supported by unrestricted grants from the Stanley Medical Research Institute (Bethesda, USA) and the Spanish Ministry of Health, Instituto de Salud Carlos III Fondos de Investigacion Sanitaria y CIBER de Salud Mental.

\section{References}

1 Colom F, Vieta E. A perspective on the use of psychoeducation, cognitivebehavioral therapy and interpersonal therapy for bipolar patients. Bipolar Disord 2004; 6: 480-6.

2 Scott J, Colom F, vieta E. A meta-analysis of relapse rates with adjunctive psychological therapies compared to usual psychiatric treatment for bipolar disorders. Int J Neuropsychopharmacol 2007; 10: 123-9.

3 Colom F, Vieta E, Martinez-Aran A, Reinares M, Goikolea JM, Benabarre A, et al. A randomized trial on the efficacy of group psychoeducation in the prophylaxis of recurrences in bipolar patients whose disease is in remission. Arch Gen Psychiatry 2003; 60: 402-7.

4 Colom F, Vieta E, Sanchez-Moreno J, Martinez-Aran A, Reinares M, Goikolea $J M$, et al. Stabilizing the stabilizer: group psychoeducation enhances the stability of serum lithium levels. Bipolar Disord 2005; 7 (suppl 5): 32-6.

5 Lam DH, Watkins ER, Hayward P, Bright J, Wright K, Kerr N, et al. A randomized controlled study of cognitive therapy for relapse prevention for bipolar affective disorder: outcome of the first year. Arch Gen Psychiatry 2003; 60: 145-52.

6 Miklowitz DJ, George EL, Richards JA, Simoneau TL, Suddath RL. A randomized study of family-focused psychoeducation and pharmacotherapy in the outpatient management of bipolar disorder. Arch Gen Psychiatry 2003; 60: 904-12.

7 Frank E, Kupfer DJ, Thase ME, Mallinger AG, Swartz HA, Fagiolini AM, et al. Two year outcomes for interpersonal and social rhythm therapy in individuals with bipolar I disorder. Arch Gen Psychiatry 2005; 62: 996-1004.

8 Simon GE, Ludman EJ, Bauer MS, Unutzer J, Operskalski B. Long-term effectiveness and cost of a systematic care program for bipolar disorder. Arch Gen Psychiatry 2006; 63: 500-8.
9 American Psychiatric Association. Diagnostic and Statistical Manual of Mental Disorder (4th edn) (DSM-IV). APA, 1994.

10 Young RC, Biggs JT, Ziegler VE, Meyer DA. A rating scale for mania: reliability, validity and sensitivity. Br J Psychiatry 1978; 133: 429-35.

11 Hamilton M. Development of a rating scale for primary depressive illness. Br J Soc Clin Psychol 1967; 6: 278-96.

12 First MB, Spitzer RL, Gibbon M, Williams JBW. Structured Clinical Interview for DSM-IV Axis I Disorders (SCID-I). American Psychiatric Press, 1994.

13 Colom F, Vieta E. Psychoeducation Manual for Bipolar Disorder. Cambridge University Press, 2006.

14 First MB, Gibbon M, Spitzer RL, Williams JBW. Structured Clinical Interview for DSM-IV Axis II Disorders (SCID-II). American Psychiatric Press, 1997.

15 Holmes TH, Rahe RH. The Social Readjustment Rating Scale. J Psychosom Res 1967; 11: 213-8.

16 Lam DH, Hayward P, Watkins ER, Wright K, Sham P. Relapse prevention in patients with bipolar disorder: cognitive therapy outcome after 2 years. Am J Psychiatry 2005; 162: 324-9.

17 Judd LL, Akiskal HS, Schettler PJ, Coryell W, Endicott J, Maser JD, et al. A prospective investigation of the natural history of the long-term weekly symptomatic status of bipolar II disorder. Arch Gen Psychiatry 2003; 60: 261-9.

18 Judd LL, Akiskal HS, Schettler PJ, Endicott J, Maser J, Solomon DA, et al. The long-term natural history of the weekly symptomatic status of bipolar I disorder. Arch Gen Psychiatry 2002; 59: 530-7.

19 Perry A, Tarrier N, Morriss R, McCarthy E, Limb K. Randomised controlled trial of efficacy of teaching patients with bipolar disorder to identify early symptoms of relapse and obtain treatment. BMJ 1999; 318: 149-53.

20 Colom F, Vieta E, Martinez-Aran A, Reinares M, Benabarre A, Gasto C. Clinical factors associated with treatment nonadherence in euthymic bipolar patients. J Clin Psychiatry 2000; 61: 549-55.

21 Colom F, Vieta E, Reinares M, Martinez-Aran A, Torrent C, Goikolea JM, et al. Psychoeducation efficacy in bipolar disorders: beyond adherence enhancement. J Clin Psychiatry 2003; 64: 1101-5.

22 Wehr TA. Improvement of depression and triggering of mania by sleep deprivation. JAMA 1992; 267: 548-51.

23 Vieta E. The package of care for patients with bipolar depression. J Clin Psychiatry 2005; 66 (suppl 5): 34-9.

24 Colom F, Vieta E, Sanchez-Moreno J, Martinez-Aran A, Torrent C, Reinares M, et al. Psychoeducation in bipolar patients with comorbid personality disorders. Bipolar Disord 2004; 6: 294-8.

25 Scott J, Paykel E, Morriss R, Bentall R, Kinderman P, Johnson T, et al. Cognitive-behavioural therapy for severe and recurrent bipolar disorders: randomised controlled trial. Br J Psychiatry 2006; 188: 313-20.

26 Reinares M, Vieta E, Colom F, Martinez-Aran A, Torrent C, Comes M, et al Impact of a psychoeducational family intervention on caregivers of stabilized bipolar patients. Psychother Psychosom 2004; 73: 312-9. 